\title{
PHOTOCHEMISTRY SIMULATION OF PLANETARY ATMOSPHERE USING SYNCHROTRON RADIATION AT SOLEIL. APPLICATION TO TITAN'S ATMOSPHERE
}

\author{
T. Gautier ${ }^{1}$, Z. Peng ${ }^{1,2}$, A. Giuliani ${ }^{3,4}$, N. Carrasco ${ }^{1}$, G. Cernogora ${ }^{1}$, \\ A. Mahjoub ${ }^{1}$, J.-J. Correia ${ }^{1}$, C. Szopa ${ }^{1}$, P. Pernot ${ }^{2}$, A. Buch ${ }^{5}$ \\ and Y. Benilan ${ }^{6}$
}

\begin{abstract}
We report here on the coupling of a gas reactor with a VUV beamline at the SOLEIL synchrotron radiation facility. The reactor may be irradiated window-less with gas pressure up to the atmosphere. The photochemistry is monitored by a mass spectrometer gas analyzer. This set up, termed APSIS for Atmospheric Photochemistry SImulated by Synchrotron, has been used to simulate the atmosphere of Titan and to study the formation of the photochemical smog and the formation of tholins.
\end{abstract}

\section{Introduction}

Laboratory simulations of photochemistry occurring in planetary atmospheres are based on photon irradiation of specific gas mixtures under temperature and pressure conditions precisely chosen to model the phenomenon under interest. Several simulations of planetary atmospheres photochemistry have been reported using laboratory photon sources (Trainer et al. 2006; Hudson et al. 2004; Gazeau et al. 2000). Most of the photon sources used in laboratory simulations (Trainer et al. 2006; Hudson et al. 2004; Gazeau et al. 2000) are chosen to deliver Lyman $\alpha$ photons, owing to the large abundance of this line in the solar spectrum. Recently,

\footnotetext{
1 Laboratoire Atmosphères, Milieux, Observations Spatiales (LATMOS), UVSQ-UPMCCNRS, 11 Bd. d'Alembert, 78280 Guyancourt, France

${ }^{2}$ Laboratoire de Chimie Physique, Université de Paris-Sud, Bât. 349, 91405 Orsay, France

${ }^{3}$ Synchrotron SOLEIL, L'Orme des Merisiers, 91192, Gif-sur-Yvette Cedex, France

${ }^{4}$ UAR1008 CEPIA, INRA, Rue de la Géraudière, 44316 Nantes, France

${ }^{5}$ Laboratoire de Génie des Procédés et Matériaux, École Centrale Paris, Grande Voie des Vignes, 92295 Châtenay Malabry Cedex, France

${ }^{6}$ Laboratoire Inter-universitaire des Systèmes Atmosphériques (LISA), 61 avenue du Général de Gaulle, 94010 Créteil Cedex, France
} 
planetary simulations have been reported using synchrotron radiation (Imanaka et al. 2009; Imanka et al. 2010). Indeed, synchrotron radiation is a very bright and broadband light source. More important than the photon flux, brightness is defined as the flux normalized to unit area and solid angle. In combination with monochromators, synchrotron radiation is an extremely useful tool to access to the ultra-violet (UV) and soft X-rays. This wide tunability overlaps perfectly the solar spectrum (Pilling et al. 2009). Indeed, comparison of the solar flux with energy integrated photon flux delivered by beamlines has allowed Pilling et al. (2009) to estimate that the irradiation process was corresponding to $10^{5}$ years of solar soft-X rays exposure on the basis of the comparison of the integrated photon fluxes for a synchrotron beamline and the sun.

In previous studies, the reaction cells, filled with a particular gas mixture, were sealed with windows transparent to the radiation. Although in that case the gas pressure may be adjusted to virtually any value, the photon energy spectrum delivered to the sample is of course limited by the transparency of the cells windows. The photon energy spectrum delivered by the source is also of importance. In cases where simulations require irradiation of the reaction cell using energetic vacuum-UV (VUV) photons, the transparency of the windows may become a serious limitation. Indeed, typical leak-tight windows made of $\mathrm{LiF}, \mathrm{CaF}_{2}$ or $\mathrm{MgF}_{2}$, which are capable of handling the pressure difference between the UHV of the beamline and the pressure of the reactor, are not transparent below $\sim 110 \mathrm{~nm}$ $(11.3 \mathrm{eV})$ at best $(\mathrm{LiF})$. Although it is relatively easy to irradiate any media with photons through such windows, it becomes more difficult to deliver to gaseous sample VUV photons of energy above the transparency limit of the windows. For such experiments, differential pumping stages have to accomodate the pressure difference between the gas reactor to be irradiated and the synchrotron beamlines, which are maintained under ultra high vacuum (UHV). These difficulties are very likely the reason why limited pressure ranges have been studied in the only previous modelisation of Titan's atmosphere using synchrotron radiation (Imanaka et al. 2009; Imanka et al. 2010).

Here we report on a new reactor, named APSIS for Atmospheric Photochemistry SImulated by Synchrotron, which is mounted on DISCO (Giuliani et al. 2009), a VUV beamline at the SOLEIL synchrotron radiation facility. This particular beamline possesses a powerful differential pumping stage (Giuliani et al. 2011) allowing us to work in a wide range of pressure up to the atmosphere and especially in the mbar range. This pressure range is comparable to that reached in other simulation methods based on plasma discharges (Szopa et al. 2006). Several experimental techniques have been implemented to simulate globally Titan's ionospheric chemistry (Imanka et al. 2010; Szopa et al. 2006). Titan, the biggest satellite of Saturn, is an important object to study, since it represents a possible analog of a frozen primitive Earth (Cable et al. 2012). Its dense atmosphere of 1.5 bars at the surface is mainly composed of nitrogen and methane. Despite the low temperature of about $200 \mathrm{~K}$, the upper atmosphere (ionosphere) undergoes efficient reaction channels, initiated by the Solar light flux and Saturn magnetospheric electrons. Complex organic matter made of nitrogenized hydrocarbons is 
produced in the gas phase leading to a brownish photochemical fog surrounding the whole satellite. The mechanisms of production of this matter are still not well understood and motivate a large spectrum of research activities from in-situ observations by instruments aboard Cassini orbiter (Waite et al. 2005) to microphysics models (Lavvas et al. 2009) and experimental simulations that mimic their production pathways in the laboratory. The key point in experimental simulation is the finding of a mean to transfer the right amount of energy to the relevant medium in terms of composition, pressure, etc. The most efficient methods known to date involve plasma techniques. Such a plasma device, named PAMPRE, has been developed in LATMOS, using a RF plasma discharge $(13.6 \mathrm{MHz})$ in a stationary flux of nitrogen and methane gas mixtures (Szopa et al. 2006; Sciamma-O'Brien et al. 2010). Plasma reactors rely on electron collision with the reactive gas background to trigger chemical reactions. Electron impact is not totally equivalent to photodissociation and photoionization in terms of cross section and reaction branching ratios. Especially, electrons may attach to molecules and lead to dissociative electron attachment (Schulz 1973) with production of negative ions, which has no equivalent under photon interaction conditions. Nevertheless, electrons are extremely convenient to deposit wide spectra of energy, even in relatively dense media. Finding equivalent energy spectrum is the major difficulty for photon-based simulations. Both the gas phase chemistries triggered by electrons in the PAMPRE plasma device and by photons in the APSIS reactor can now be compared. The validity of the plasma approach will be explored. Finally, this APSIS set-up may be used to study the gas phase chemistry at work when nitrogen and methane mixture are irradiated for different pressures, thus reflecting various altitudes in Titan's atmosphere.

\section{The APSIS set up}

The DISCO beamline is a bending magnet based beamline and has been described in more details elsewhere (Giuliani et al. 2009). It possesses three end-stations. One of those, referred to as APEX (for Atmospheric Pressure Experiment) is fitted with an atmospheric pressure differential pumping system, which allows delivering monochromatic VUV photons down to $60 \mathrm{~nm}$ in rare gas atmospheres (Giuliani et al. 2011). The reactor was mounted on the APEX port. The principle of the set up is shown in Figure 1. The reactor is made of a stainless steel chamber fitted with a gas inlet from which the reactive mixture is introduced. A gas flow meter (10 sccm MKS model) controls the flow rate of the nitrogen - methane mixture (90\% - 10\%). A RGA, residual gas analyzer (Pfeiffer QME 200 quadrupole mass spectrometer) is connected to a moveable nose, and analyzes in line the composition of the gas mixture inside the reactor. The white beam from the beamline is sent inside the reactor. Between the beamline and the chamber a T-shape pipe allows introducing a flow of helium. This gas is used to avoid pumping the reactive mixture by the differential pumping system and thus confines the reactive mixture inside the reactor. The photochemistry thus occurs only inside the reactor. The pressure of the helium curtain has to be adjusted for each mixture pressure 


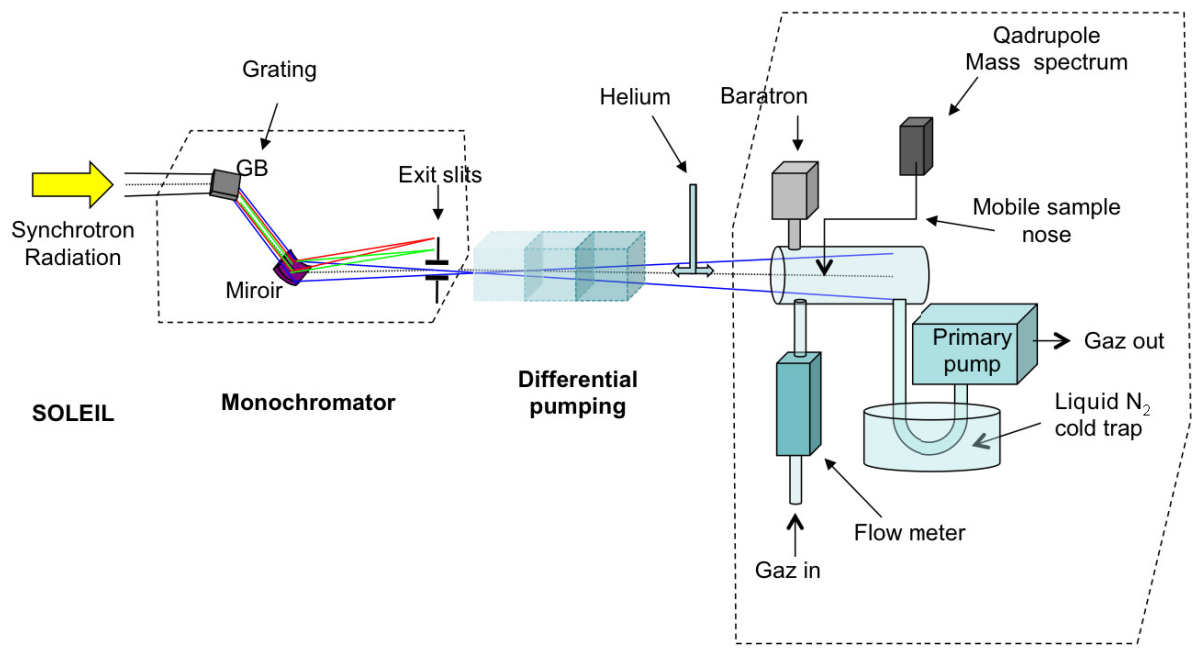

APSIS reactor

Fig. 1. Principle of the Atmospheric Photochemistry SImulated by Synchrotron (APSIS) set up and its coupling with the APEX branch of the DISCO beamline.

in the reactor. First, the reactor is evacuated using a turbomolecular pump. Then the mixture is admitted inside at flow rates around $7 \mathrm{sccm}$ of nitrogen methane mixture. The pressure inside the reactor, as monitored by a capacitance gauge, is 10 mbar. Helium is admitted and the pressure rises inside the reactor up to 15 mbar. A dry pump evacuates the reactor in order to establish a flow. Nevertheless, the gas flow has to be low enough to provide enough time to the reactive species to react. For this, the conductance upstream the pump has been reduced using particulate filters (Teflon filters with pores of $100 \mathrm{~nm}$ ). It is possible to insert between the pump and the chamber a cold trap cooled using liquid nitrogen to condense any low abundant photochemical product, that can then be analyzed ex-situ (Gautier et al. 2011). Owing to the helium curtain, the photon spectrum is limited by the ionization energy of helium $24.58 \mathrm{eV}$ (Rosenstock et al. 2011). Other curtain gases may be used instead of helium, such as neon or krypton, to narrow the photon energy window. Once the gas flow is established, the mixture is kept nonirradiated and a background spectrum is recorded with the RGA. This obtained mass spectrum serves as a reference to assess any production (increase of ionic abundance) or consumption (decrease in abundance) of any molecular species.

\section{Conclusions}

We report here the first results from the coupling of the VUV synchrotron beamline with a window less gas reactor, referred to as APSIS, able to simulate all kind of atmospheric composition and pressure. The system has been fed with a nitrogen 
methane mixture found on Titan. Irradiation of the reactor with VUV photons has resulted in the production of photochemical species, such as $H C N$, propane $\left(\mathrm{C}_{3} \mathrm{H}_{8}\right)$, acetonitrile $\left(\mathrm{CH}_{3} \mathrm{CN}\right)$ and cyanogen $\left(\mathrm{C}_{2} \mathrm{~N}_{2}\right)$, which are suggested to account for the formation of the Titan photochemical fog (Carrasco et al. 2012).

\section{References}

Cable, M.L., Hrst, S.M., Hodyss, R., et al., 2012, Chem. Rev., 112, 1882

Carrasco, N., Gautier, T., Es-sebbar, Et-T., Pernot, P., \& Cernogora, G., 2012, Icarus, in press

Gautier, T., Carrasco, N., Buch, A., Szopa, C., Sciamma-O'Brien, E., \& Cernogora, G., 2011, Icarus, 213, 625

Gazeau, M., Cottin, H., Vuitton, V., Smith, N., \& Raulin, F., 2000, Planet. Space Sci., 48,437

Giuliani, A., Jamme, F., Rouam, V., et al., 2009, J. Synchrotron Rad., 16, 835

Giuliani, A., Yao, I., Lagarde, B., et al., 2011, J. Synchrotron Rad., 18, 546

Hudson, R.L., \& Moore, M., 2004, Icarus, 172, 466

Imanaka, H., \& Smith, M.A., 2009, J. Phys. Chem. A, 113, 11187

Imanaka, H., \& Smith, M.A., 2010, PNAS, 107, 12423

Lavvas, P., et al., 2009, Icarus, 201, 626

Pilling, S., Andrade, D.P.P., Neto, A.C., Rittner, R., \& Naves de Brito, A., 2009, J. Phys. Chem. A, 113, 11161

Rosenstock, H.M., Draxl, K., \& Steiner, B.W., 2011, in "Ion Energetics Data", NIST Chemistry WebBook, NIST database and standard reference, number 69, ed. P.J. Linstrom \& W.G. Mallard, National Institute of Standards and Technology, Gaithersburg MD (December 2011)

Schulz, G.J., 1973, Rev. Mod. Phys., 45, 423

Sciamma-O'Brien, E., Carrasco, N., Szopa, C., Buch, A., \& Cernogora, G., 2010, Icarus, 209, 704

Szopa, C., Cernogora, G., Boufendi, L., Correia, J.-J., \& Coll, P., 2006, Planet. Space Sci., 54, 394

Trainer, M.G., Pavlov, A.A., DeWitt, H.L., et al., 2006, PNAS, 103, 18035

Waite Jr., J.H., et al., 2005, Science, 308, 982 